\title{
HAAR-TYPE MULTIWAVELET BASES AND SELF-AFFINE MULTI-TILES*
}

\author{
TIM FLAHERTY† AND YANG WANG W $^{\dagger}$
}

\begin{abstract}
Gröchenig and Madych showed that a Haar-type wavelet basis of $L^{2}\left(\mathbf{R}^{n}\right)$ can be constructed from the characteristic function $\chi_{\Omega}$ of a compact set $\Omega$ if and only if $\Omega$ is an integral self-affine tile of Lebesgue measure one. In this paper we generalize their result to the multiwavelet settings. We give a complete characterization of Haar-type scaling function vectors $\chi_{\Omega}(x):=\left[\chi_{\Omega_{1}}(x), \ldots, \chi_{\Omega_{r}}(x)\right]^{T}$, where $\Omega=\left(\Omega_{1}, \ldots, \Omega_{r}\right)$ is an $r$-tuple of compact sets in $\mathbf{R}^{n}$. We call $\Omega$ a self-affine multi-tile because $\Omega_{i}$ 's tile $\mathbf{R}^{n}$ by translation and have the property that each affine image $A\left(\Omega_{i}\right)$ is the union of translates of some $\Omega_{j}$ 's. We also construct associated Haar-type multiwavelets, and present examples using various dilation matrices $A$.
\end{abstract}

1. Introduction. Let $A$ be an expanding matrix in $M_{n}(\mathbf{Z})$, that is, one with integer entries and all eigenvalues $\left|\lambda_{i}(A)\right|>1$. A compactly supported nonzero function $f(x) \in L^{2}\left(\mathbf{R}^{n}\right)$ is called a scaling function of a multiresolution analysis with dilation factor $A$ if it has the following properties:

(i) $f(x)$ satisfies a refinement equation

$$
f(x)=\sum_{\alpha \in \mathbf{Z}^{n}} c_{\alpha} f(A x-\alpha)
$$

where the coefficients $c_{\alpha}$ are real and $\sum_{\alpha \in \mathbf{Z}^{n}} c_{\alpha}=|\operatorname{det}(A)|$.

(ii) The integer translates $f(x-\alpha), \alpha \in \mathbf{Z}^{n}$, are orthogonal in $L^{2}\left(\mathbf{R}^{n}\right)$.

It is well known that given any scaling function of a multiresolution analysis, an orthonormal wavelet basis can be constructed from that scaling function, see [5].

In [13] Gröchenig and Madych studied Haar-type scaling functions and wavelet bases. A Haar-type scaling function is a scaling function of the form $\chi_{\Omega}$, where $\Omega$ is a compact set in $\mathbf{R}^{n}$, and a Haar-type wavelet basis is the wavelet basis constructed from a Haar-type scaling function. Gröchenig and Madych showed that for a given expanding matrix $A \in M_{n}(\mathbf{R})$ the function $\chi_{\Omega}$ is a scaling function of a multiresolution analysis with dilation $A$ if and only if $\Omega$ satisfies the following conditions:

(i) $\Omega$ is a $\mathbf{Z}^{n}$-tile of $\mathbf{R}^{n}$, i.e.,

$$
\bigcup_{\alpha \in \mathbf{Z}^{n}}(\Omega+\alpha)=\mathbf{R}^{n}
$$

and the union is measure disjoint.

(ii) $\Omega$ satisfies a set-valued equation (up to a measure zero set)

$$
A(\Omega)=\bigcup_{d \in \mathcal{D}}(\Omega+d)
$$

for some finite digit set $\mathcal{D} \in \mathbf{Z}^{n}$ of cardinality $|\operatorname{det}(A)|$.

\footnotetext{
* Received September 23, 1998; accepted for publication December 8, 1998.

${ }^{\dagger}$ Mathematical Sciences Department, Carnegie Mellon University, Pittsburgh, PA 15213, USA (tim@andrew.cmu.edu).

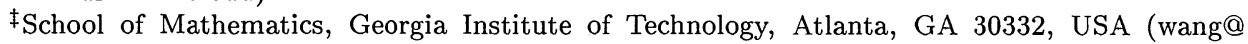
math.gatech.edu). Research supported in part by the National Science Foundation, grant DMS9706793.
} 
We call a compact set $\Omega$ with positive Lebesgue measure that satisfies the condition (ii) a self-affine tile. It is known that such an $\Omega$ must have nonempty interior, and must tile $\mathbf{R}^{n}$ by translation by some subset of $\mathbf{Z}^{n}$. If in addition $\Omega$ is a $\mathbf{Z}^{n}$-tile of $\mathbf{R}^{n}$, then the digit set $\mathcal{D}$ must form a complete residue system $(\bmod A)$, i.e., a complete set of coset representatives of the group $\mathbf{Z}^{n} / A\left(\mathbf{Z}^{n}\right)$. Conversely, if the digit set $\mathcal{D}$ is a complete residue system $(\bmod A)$ then there is a unique compact set $\Omega$ satisfying (1.2), and $\Omega$ must be a self-affine tile, namely it must have positive Lebesgue measure. Note that there is an obviously equivalent formulation of (1.2) in the form of a refinement equation, which is

$$
\chi_{\Omega}(x)=\sum_{d \in \mathcal{D}} \chi_{\Omega}(A x-d)
$$

Self-affine tiles have been extensively studied in recent years. More results on them can be found in Bandt [1], Gröchenig and Haas [11], Kenyon [18], and Lagarias and Wang [23], [19], [20].

In this paper we study Haar-type scaling function vectors and multiwavelet bases. Scaling function vectors are generalizations of scaling functions to vector valued functions. More precisely, a vector valued function $f(x)=\left[f_{1}(x), \ldots, f_{r}(x)\right]^{T}$, with each compactly supported $f_{i}(x) \in L^{2}\left(\mathbf{R}^{n}\right)$, is a scaling function vector if the following two conditions are met:

(a) $f(x)$ satisfies a vector refinement equation

$$
f(x)=\sum_{\alpha \in \mathbf{Z}^{n}} c_{\alpha} f(A x-\alpha),
$$

where the coefficients $c_{\alpha}$ are matrices in $M_{r}(\mathbf{R}) \cdot r$ is called the vector multiplicity of $f(x)$.

(b) $v_{f}:=\int_{\mathbf{R}^{n}} f(x) d x \neq 0$ and for all $\alpha, \beta \in \mathbf{Z}^{n}$,

$$
\int_{\mathbb{R}^{n}} f(x-\alpha) f^{T}(x-\beta) d x=\delta_{\alpha-\beta} \Lambda
$$

where $\Lambda$ is an $r \times r$ diagonal matrix with positive diagonal elements and $\delta_{\gamma}$ is the standard Kronecker symbol with $\delta_{0}=1$ and $\delta_{\gamma}=0$ otherwise.

Note that $v_{f} \neq 0$ implies that $v_{f}$ is a $|\operatorname{det}(A)|$-eigenvector of the matrix $c:=$ $\sum_{\alpha \in \mathbf{Z}^{n}} c_{\alpha}$.

As with the case of a scaling function, an orthonormal basis of $L^{2}\left(\mathbf{R}^{n}\right)$ called a multiwavelet basis can be constructed from a scaling function vector. Multiwavelets have received considerable attention recently, after the construction of multiwavelets by Donovan, Geronimo, Hardin, Kessler and Massopust using fractal interpolation functions [15], [9], [6], and by Goodman and Lee [10]. One advantage of multiwavelets is that they can be made to combine smoothness with small supports. Although these properties are achieved at the cost of using more wavelets, their potential importance in numerical applications may outweight the cost.

Definition 1.1. We say a function vector $f: \mathbf{R}^{n} \rightarrow \mathbf{R}^{r}$ is of Haar-type if each component of $f(x)$ is the characteristic function of some compact set.

Now, let $\boldsymbol{\Omega}=\left(\Omega_{1}, \ldots, \Omega_{r}\right)$ be an $r$-tuple of compact sets in $\mathbf{R}^{n}$, and let $\chi_{\boldsymbol{\Omega}}(x):=$ $\left[\chi_{\Omega_{1}}(x), \ldots, \chi_{\Omega_{r}}(x)\right]^{T}$. It is then natural to ask whether $\chi_{\Omega}(x)$ is a scaling function vector for some given dilation $A$. Our main theorems, stated below, classify all Haartype scaling function vectors. 
Theorem 1.1. Let $A \in M_{n}(\mathbf{Z})$ be an expanding matrix and $\boldsymbol{\Omega}=\left(\Omega_{1}, \ldots, \Omega_{r}\right)$ be an $r$-tuple of compact sets in $\mathbf{R}^{n}$. Suppose that $\chi_{\Omega}(x)$ is a scaling function vector satisfying the vector refinement equation

$$
\chi_{\Omega}(x)=\sum_{\alpha \in \mathbf{Z}^{n}} c_{\alpha} \chi_{\Omega}(A x-\alpha) .
$$

Then

(i) Each coefficient matrix $c_{\alpha} \in M_{r}(\mathbf{R})$ is a zero-one matrix.

(ii) For each $\alpha \in \mathbf{Z}^{n}$ the matrix

$$
b_{\alpha}=\sum_{\beta \in \mathbf{Z}^{n}} c_{\alpha+A \beta}
$$

is a zero-one matrix and contains exactly one entry of 1 in each column.

(iii) The nonnegative matrix $c=\sum_{\alpha \in \mathbf{Z}^{n}} c_{\alpha}$ is irreducible.

(iii') The nonnegative matrix $c=\sum_{\alpha \in \mathbf{Z}^{n}} c_{\alpha}$ is primitive. ${ }^{1}$

(iv) $\left\{\Omega_{i}: 1 \leq i \leq r\right\}$ are measure disjoint, and $\cup_{i=1}^{r} \Omega_{i}$ is a $\mathbf{Z}^{n}$-tile of $\mathbf{R}^{n}$.

We use the term self-affine multi-tile for $\Omega$ because $\mathbf{R}^{n}$ can be tiled by the translates of $\Omega_{1}, \ldots, \Omega_{r}$, and for all $1 \leq i \leq r$ we have

$$
A\left(\Omega_{i}\right)=\bigcup_{j=1}^{r}\left(\Omega_{j}+\mathcal{D}_{i j}\right)
$$

where each $\mathcal{D}_{i j}$ is a finite, possibly empty, set in $\mathbf{Z}^{n}$, with all unions in (1.7) measure disjoint.

We will now briefly describe the construction of Haar-type multiwavelet bases which correspond to the scaling function vectors of Theorem 1.1. Let $f=\chi_{\Omega}$ be a scaling function vector satisfying (1.6). Let $\mathcal{E}_{i}=\left\{(\alpha, j):\left(c_{\alpha}\right)_{i j}=1\right\}$ for all $1 \leq i \leq r$, and set $\gamma_{i}=\left|\mathcal{E}_{i}\right|$. For each $i$ define an arbitrary bijection $I_{i}$ mapping $\mathcal{E}_{i}$ onto $\left\{1,2, \ldots, \gamma_{i}\right\}$. We now normalize the scaling functions, setting $\phi_{i}=\left\|f_{i}\right\|_{2}^{-1} f_{i}$. Next let $M_{i}$ be an orthogonal $\gamma_{i} \times \gamma_{i}$ matrix with the first row $\gamma_{i}^{-1 / 2}[1,1, \ldots, 1]$. Define, for each $1 \leq i \leq r$, and $2 \leq j \leq \gamma_{i}$ the following functions

$$
g_{i j}(x)=|\operatorname{det}(A)| \sum_{(\alpha, k) \in \mathcal{E}_{i}} M_{i}\left(j, I_{i}(\alpha, k)\right) \phi_{k}(A x-\alpha) .
$$

We have that $g_{i j}$ is supported on the set $\Omega_{i}$, using (1.6). Hence, we only need to establish orthonormality of $g_{i j}$ to $g_{i k}$, and of $g_{i j}$ to $\phi_{k}$. This readily follows by computing these inner products, applying the refinement equations, and using the orthogonality of the $M_{i}$. Finally, observe that we have a total of $\gamma_{1}+\cdots+\gamma_{r}-r=|\operatorname{det}(A)| r-r$ wavelet functions, precisely the number of wavelets needed.

Theorem 1.2. Let $A \in M_{n}(\mathbf{Z})$ be an expanding matrix. Suppose that the coefficient matrices $c_{\alpha}$ of the vector refinement equation

$$
f(x)=\sum_{\alpha \in \mathbf{Z}^{n}} c_{\alpha} f(A x-\alpha)
$$

satisfy properties (i)-(iii) of Theorem 1.1. Then up to scalar multiplications the vector refinement equation has a unique compactly supported solution, which is of Haar-type $f(x)=\chi_{\Omega}(x)$. Furthermore, $\boldsymbol{\Omega}=\left(\Omega_{1}, \ldots, \Omega_{r}\right)$ satisfies

\footnotetext{
${ }^{1}$ Note that (iii') is stronger than (iii). We include (iii) to simplify the statement of Theorem 1.2.
} 
(A) Each $\Omega_{i}$ has nonempty interior, and $\Omega_{i}=\overline{\Omega_{i}^{o}}$.

(B) There exist subsets $\mathcal{J}_{1}, \ldots, \mathcal{J}_{r}$ of $\mathbf{Z}^{n}$ such that

$$
\bigcup_{i=1}^{r} \bigcup_{\gamma_{i} \in \mathcal{J}_{i}}\left(\Omega_{i}+\gamma_{i}\right)
$$

is a tiling of $\mathbf{R}^{n}$.

We remark that the solution $\chi_{\Omega}$ in Theorem 1.2 is not necessarily a scaling function vector, even when $n=1$ and $r=1$. The simplest counter-example is the refinement equation

$$
f(x)=f(2 x)+f(2 x-3),
$$

which admits the Haar-type solution $f(x)=\chi_{[0,3)}(x)$ that is not a scaling function. Checking whether a solution $\chi_{\Omega}$ is a scaling function vector can be done in finitely many steps using an appropriate generalization of an algorithm in Lawton [24]. For a solution $\chi_{\Omega}$ that is not a scaling function vector, it is not known whether $\cup_{i=1}^{r} \Omega_{i}$ still tiles $\mathbf{R}^{n}$ by translation. We state here as a conjecture that it does.

The rest of this paper are organized as follows: In $\S 2$ we state and prove some general results concerning scaling function vectors and certain generalization of iterated function systems. We then use these results to prove our main theorems in $\S 3$. In $\S 4$ we present some examples of self-affine multi-tiles.

The second author would like to thank Professor Ka-Sing Lau for the invitation to visit the Chinese University of Hong Kong, where this research was conducted primarily. Both authors thank the mathematics department of CUHK for the generous support, and Professor Lau and Dr. Sze-Man Ngai in particular for their hospitality and stimulating discussions. After we completed this paper we received a preprint by Gröchenig, Haas and Raugi [12] that contains some overlapping results.

2. General Results. Consider the vector refinement equation

$$
f(x)=\sum_{\alpha \in \mathbf{Z}^{n}} c_{\alpha} f(A x-\alpha)
$$

where $f(x)=\left[f_{1}(x), \ldots, f_{r}(x)\right]^{T}, c_{\alpha} \in M_{r}(\mathbf{R})$ and $c_{\alpha} \neq 0$ for only finitely many $\alpha$. We define the symbol of the vector refinement equation to be

$$
m(\xi)=\frac{1}{|\operatorname{det}(A)|} \sum_{\alpha \in \mathbf{Z}^{n}} c_{\alpha} e^{-i\langle\alpha, \xi\rangle} .
$$

Let $B:=A^{T}$. It verifies easily that the Fourier transform of $f(x)$ satisfies

$$
\widehat{f}(\xi)=m\left(B^{-1} \xi\right) \widehat{f}\left(B^{-1} \xi\right) .
$$

Some standard properties for non-vector refinement equations are known to generalize to the vector refinement equation (2.1). For example, if $f(x)$ is integrable and $v_{f}:=$ $\int_{\mathbf{R}^{n}} f(x) d x \neq 0$ then $v_{f}$ is a 1-eigenvector of the matrix $m(0)$. But unlike the nonvector case, a given vector refinement equation may have more than one independent

\footnotetext{
${ }^{2}$ If we interpret the equality in (1.9) as almost everywhere, then $\Omega_{i}=\overline{\Omega_{i}^{o}}$ up to a measure zero set.
} 
compactly supported $L^{1}$ solution. In fact, under the assumption that the spectral radius $\rho(m(0)) \leq 1$ each 1-eigenvector $v$ of $m(0)$ leads to a compactly supported solution $f(x)$ (in the sense of tempered distribution) to the vector refinement equation (2.1) via

$$
\widehat{f}(\xi)=\left(\prod_{j=1}^{\infty} m\left(B^{-j} \xi\right)\right) v,
$$

see Cohen, Daubechies and Plonka [4].

For a vector valued function $f(x)$ we define

$$
v_{f}:=\int_{\mathbf{R}^{n}} f(x) d x, \quad \Lambda_{f}:=\int_{\mathbf{R}^{n}} f(x) f^{T}(x) d x .
$$

For a scaling function vector $f(x)$ the matrix $\Lambda_{f}$ is a diagonal matrix with positive diagonal entries.

Lemma 2.1. Suppose that $f(x)$ satisfies (2.1) and is a scaling function vector. Then

$$
\sum_{\alpha \in \mathbf{Z}^{n}} c_{\alpha} \Lambda_{f} c_{\alpha+A \beta}^{T}=\delta_{\beta}|\operatorname{det}(A)| \Lambda_{f}
$$

Proof. Let $q=|\operatorname{det}(A)|$. By definition of a scaling function vector,

$$
\begin{aligned}
\delta_{\beta}|\operatorname{det}(A)| \Lambda_{f} & =q \int_{\mathbf{R}^{n}} f(x) f^{T}(x+\beta) d x \\
& =q \sum_{\alpha \in \mathbf{Z}^{n}} \sum_{\gamma \in \mathbf{Z}^{n}} c_{\alpha}\left(\int_{\mathbf{R}^{n}} f(A x-\alpha) f^{T}(A x+A \beta-\gamma) d x\right) c_{\gamma}^{T} \\
& =\sum_{\alpha \in \mathbf{Z}^{n}} \sum_{\gamma \in \mathbf{Z}^{n}} c_{\alpha}\left(\int_{\mathbf{R}^{n}} f(y-\alpha) f^{T}(y+A \beta-\gamma) d y\right) c_{\gamma}^{T} \\
& =\sum_{\alpha \in \mathbf{Z}^{n}} c_{\alpha}\left(\int_{\mathbf{R}^{n}} f(y-\alpha) f^{T}(y-\alpha) d y\right) c_{\alpha+A \beta}^{T} \\
& =\sum_{\alpha \in \mathbf{Z}^{n}} c_{\alpha} \Lambda_{f} c_{\alpha+A \beta}^{T} .
\end{aligned}
$$

Definition 2.1. Let $\Lambda$ be a diagonal matrix with positive diagonal entries. The vector refinement equation (2.1) is said to satisfy the orthogonal coefficients condition (with respect to $\Lambda$ ) if

$$
\sum_{\alpha \in \mathbf{Z}^{n}} c_{\alpha} \Lambda c_{\alpha+A \beta}^{T}=\delta_{\beta}|\operatorname{det}(A)| \Lambda
$$

THEOREM 2.2. Suppose that the vector refinement equation (2.1) satisfies the orthogonal coefficients condition. Suppose further that $v_{0} \in \mathbf{R}^{r}$ is a 1-eigenvector of $m(0)=|\operatorname{det}(A)|^{-1} \sum_{\alpha \in \mathbf{Z}^{n}} c_{\alpha}$, and that $\rho(m(0)) \leq 1$. Then there exists a nontrivial compactly supported $L^{2}$ solution $f(x)=\left[f_{1}(x), \ldots, f_{r}(x)\right]^{T}$ of the vector refinement equation (2.1) such that $v_{f}=v_{0}$. 
Proof. The orthogonal coefficients condition implies the existence of a diagonal matrix $\Lambda=\operatorname{diag}\left(\lambda_{1}, \ldots, \lambda_{r}\right)$ with $\lambda_{i}>0$ such that

$$
\sum_{\alpha \in \mathbf{Z}^{n}} c_{\alpha} \Lambda c_{\alpha+A \beta}^{T}=\delta_{\beta}|\operatorname{det}(A)| \Lambda
$$

We partition the cube $[0,1)^{n}$ into disjoint sets $\Omega_{1}, \ldots, \Omega_{r}$ of positive Lebesgue measures. Let $g(x)=\left[g_{1}(x), \ldots, g_{r}(x)\right]^{T}$ such that: (1) each $g_{i}(x)$ is supported on $\Omega_{i}$; (2) $v_{g}=v_{0}$; (3) $\Lambda_{g}=t \Lambda$ for some $t>0$. Without loss of generality we assume that $t=1, \Lambda_{g}=\Lambda$.

Let $\mathcal{T}$ be the operator defined by

$$
\mathcal{T} h(x)=\sum_{\alpha \in \mathbf{Z}^{n}} c_{\alpha} h(A x-\alpha) .
$$

Then $\widehat{\mathcal{T h}}(\xi)=m\left(B^{-1} \xi\right) \widehat{h}\left(B^{-1} \xi\right)$ where $B=A^{T}$. Denote $g_{k}=\mathcal{T}^{k} g$ for $k \geq 0$. By induction on $k$ it is easily verified that

$$
v_{g_{k}}=v_{0} \quad \text { and } \quad \int_{\mathbf{R}^{n}} g_{k}(x) g_{k}(x-\alpha)^{T} d x=\delta_{\alpha} \Lambda
$$

Therefore there exists a constant $K>0$ such that

$$
\int_{\mathbf{R}^{n}}\left|\widehat{g}_{k}(\xi)\right|^{2} d \xi \leq K
$$

where |.| denotes the Euclidean norm on $\mathbf{C}^{r}$. Now

$$
\widehat{g}_{k}(\xi)=\left(\prod_{j=1}^{k} m\left(B^{-j} \xi\right)\right) \widehat{g}\left(B^{-k} \xi\right)
$$

So by Theorem 3.2 of Cohen, Daubechies and Plonka [4], $\widehat{g}_{k}(\xi)$ converges unformly on compact sets to the entire function $\widehat{f}(\xi):=\left(\prod_{j=1}^{\infty} m\left(B^{-j} \xi\right)\right) v_{0}{ }^{3}$. The function $f(x)$, which is the inverse Fourier transform of $\widehat{f}(\xi)$, is compactly supported and satisfies the vector refinement equation (2.1) because $\widehat{f}(\xi)=m\left(B^{-1} \xi\right) \widehat{f}\left(B^{-1} \xi\right)$. Moreover, $f(x)$ is $L^{2}$ because $\widehat{f}(\xi)$ is. Finally, $v_{f}=\widehat{f}(0)=v_{0}$.

Our next two theorems concern certain generalization of iterated function systems (IFS). Let $\mathcal{C}_{n}$ denote the space of all nonempty compact subsets of $\mathbf{R}^{n}$. Let $\|\cdot\|$ be a norm on $\mathbf{R}^{n}$. We define the Hausdorff metric on $\mathcal{C}_{n}$ with respect to the norm by

$$
\mathrm{d}_{\mathrm{H}}\left(D, D^{\prime}\right):=\max \left\{\sup _{x \in D} \inf _{x^{\prime} \in D^{\prime}}\left\|x-x^{\prime}\right\|, \sup _{y^{\prime} \in D^{\prime}} \inf _{y \in D}\left\|y-y^{\prime}\right\|\right\} .
$$

It is well known that $\left(\mathcal{C}_{n}, \mathrm{~d}_{\mathrm{H}}\right)$ is a complete metric space. Now let $\mathrm{d}_{\mathrm{H}}^{r}$ be the metric defined on $\mathcal{C}_{n}^{r}$, the space of all $r$-tuples of nonempty compact subsets of $\mathbf{R}^{n}$, given by

$$
\mathrm{d}_{\mathrm{H}}^{r}\left(\Omega, \Omega_{1}^{\prime}\right):=\max _{1 \leq i \leq r}\left\{\mathrm{~d}_{\mathrm{H}}\left(\Omega_{1}, \Omega_{1}^{\prime}\right), \ldots, \mathrm{d}_{\mathrm{H}}\left(\Omega_{r}, \Omega_{r}^{\prime}\right)\right\}
$$

\footnotetext{
${ }^{3}$ The result was established in Theorem 3.2 for $A=2 I$. But the proof obviously generalizes to an arbitrary expanding matrix $A$.
} 
Then $\left(\mathcal{C}_{n}^{r}, \mathrm{~d}_{\mathrm{H}}^{r}\right)$ is also a complete metric space. The following result is essentially due to Mauldin and Williams [27]. We include a proof for self-containment.

Proposition 2.3. Let $A$ be an expanding matrix in $M_{n}(\mathbf{R})$ and let $\mathcal{D}_{i j}, 1 \leq$ $i, j \leq r$, be finite subsets of $\mathbf{R}^{n}$, with $\cup_{j=1}^{r} \mathcal{D}_{i j}$ nonempty. Then there exists a unique $\boldsymbol{\Omega}=\left(\Omega_{1}, \ldots, \Omega_{r}\right) \in \mathcal{C}_{n}^{r}$ such that

$$
A\left(\Omega_{i}\right)=\bigcup_{j=1}^{r}\left(\Omega_{j}+\mathcal{D}_{i j}\right), \quad 1 \leq i \leq r .
$$

Proof. Since $A$ is expanding, there exists a norm $\|\cdot\|$ on $\mathbf{R}^{n}$ such that $\left\|A^{-1} x\right\| \leq$ $\lambda\|x\|$ where $0<\lambda<1$ for all $x \in \mathbf{R}^{n}$. Let the Hausdorff metric $\mathrm{d}_{\mathbf{H}}$ be defined using this norm. We now consider the map $\Phi: \mathcal{C}_{n}^{r} \rightarrow \mathcal{C}_{n}^{r}$ defined by

$$
\Phi_{i}(\boldsymbol{\Omega})=\bigcup_{j=1}^{r}\left(A^{-1}\left(\Omega_{j}\right)+A^{-1}\left(\mathcal{D}_{i j}\right)\right), \quad 1 \leq i \leq r .
$$

We show that $\Phi$ is contractive in $\left(\mathcal{C}_{n}^{r}, \mathrm{~d}_{\mathrm{H}}^{r}\right)$. Note that

$$
\mathrm{d}_{\mathrm{H}}\left(\bigcup_{j=1}^{m} E_{j}, \bigcup_{j=1}^{m} E_{j}^{\prime}\right) \leq \max _{1 \leq j \leq m} \mathrm{~d}_{\mathrm{H}}\left(E_{j}, E_{j}^{\prime}\right)
$$

whenever $E_{j}, E_{j^{\prime}}$ are all in $\mathcal{C}_{n}$, see Barnsley [2]. Set $\Gamma_{i}=\left\{j: \mathcal{D}_{i j} \neq \emptyset\right\}$. Now for any $\boldsymbol{\Omega}, \boldsymbol{\Omega}^{\prime} \in \mathcal{C}_{n}^{r}$ we have

$$
\begin{aligned}
\mathrm{d}_{\mathrm{H}}\left(\Phi_{i}(\boldsymbol{\Omega}), \Phi_{i}\left(\boldsymbol{\Omega}^{\prime}\right)\right) & \leq \max _{j \in \Gamma_{i}} \mathrm{~d}_{\mathrm{H}}\left(A^{-1}\left(\Omega_{j}+\mathcal{D}_{i j}\right), A^{-1}\left(\Omega_{j}^{\prime}+\mathcal{D}_{i j}\right)\right) \\
& \leq \lambda \max _{j \in \Gamma_{i}} \mathrm{~d}_{\mathrm{H}}\left(\Omega_{j}+\mathcal{D}_{i j}, \Omega_{j}^{\prime}+\mathcal{D}_{i j}\right) \\
& \leq \lambda \max _{j \in \Gamma_{i}} \max _{\gamma \in \mathcal{D}_{i j}} \mathrm{~d}_{\mathrm{H}}\left(\Omega_{j}+\gamma, \Omega_{j}^{\prime}+\gamma\right) \\
& =\lambda \max _{j \in \Gamma_{i}} \mathrm{~d}_{\mathrm{H}}\left(\Omega_{j}, \Omega_{j}^{\prime}\right) \\
& \leq \lambda \max _{1 \leq j \leq r} \mathrm{~d}_{\mathrm{H}}\left(\Omega_{j}, \Omega_{j}^{\prime}\right) \\
& =\lambda \mathrm{d}_{\mathrm{H}}^{r}\left(\boldsymbol{\Omega}, \Omega^{\prime}\right) .
\end{aligned}
$$

Hence

$$
\mathrm{d}_{\mathrm{H}}^{r}\left(\Phi(\boldsymbol{\Omega}), \Phi\left(\boldsymbol{\Omega}^{\prime}\right)\right)=\max _{1 \leq i \leq r} \mathrm{~d}_{\mathrm{H}}\left(\Phi_{i}(\boldsymbol{\Omega}), \Phi_{i}\left(\boldsymbol{\Omega}^{\prime}\right)\right) \leq \lambda \mathrm{d}_{\mathrm{H}}^{r}\left(\boldsymbol{\Omega}, \boldsymbol{\Omega}^{\prime}\right) .
$$

So $\Phi$ is contractive, and it follows that there exists a unique $\Omega \in \mathcal{C}_{n}^{r}$ such that $\Phi(\boldsymbol{\Omega})=\boldsymbol{\Omega}$, proving the theorem.

We remark that the irreduciblity condition on $c=\sum_{\alpha \in \mathbf{Z}^{n}} c_{\alpha}$ implies that $\cup_{j=1}^{r} \mathcal{D}_{i j}$ is nonempty, where $\mathcal{D}_{i j}=\left\{\alpha:\left(c_{\alpha}\right)_{i j}=1\right\}$.

TheOREM 2.4. Let $A$ be an expanding matrix in $M_{n}(\mathbf{Z})$ and let $\mathcal{D}_{i j}, 1 \leq i, j \leq r$, be finite (possibly empty) subsets of $\mathbf{Z}^{n}$. Let $\boldsymbol{\Omega}=\left(\Omega_{1}, \ldots, \Omega_{r}\right) \in \mathcal{C}_{n}^{r}$ be the solution to

$$
A\left(\Omega_{i}\right)=\bigcup_{j=1}^{r}\left(\Omega_{j}+\mathcal{D}_{i j}\right), \quad 1 \leq i \leq r .
$$


Suppose that $\Omega=\cup_{i=1}^{r} \Omega_{i}$ has positive Lebesgue measure. Then $\Omega+\mathbf{Z}^{n}=\mathbf{R}^{n}$ and $\Omega$ has nonempty interior.

Proof. Let $\pi: \mathbf{R}^{n} \rightarrow \mathbf{T}^{n}:=\mathbf{R}^{n} / \mathbf{Z}^{n}$ be the canonical covering map. Denote $A_{*}:=\pi \circ A \circ \pi^{-1}$ and $\bar{\Omega}=\pi(\Omega)$. Then

$$
A_{*}(\bar{\Omega})=\bigcup_{i=1}^{r} \bigcup_{j=1}^{r} \pi\left(\Omega_{j}+\mathcal{D}_{i j}\right) \subseteq \bigcup_{i=1}^{r} \pi\left(\Omega_{i}\right)=\bar{\Omega}
$$

Note that we only have $A_{*}(\bar{\Omega}) \subseteq \bar{\Omega}$ because some of the $\mathcal{D}_{i j}$ 's may be empty. So $\bar{\Omega}$ is invariant under the ergodic map $A$ on $\mathbf{T}^{n}$. This implies that either $\mu(\bar{\Omega})=0$ or $\bar{\Omega}=\mathbf{T}^{n}$ up to a measure zero subset. But $\mu(\Omega)>0$, so $\bar{\Omega}=\mathbf{T}^{n}$. Hence $\Omega$ contains a fundamental domain of the lattice $\mathbf{Z}^{n}$, and $\Omega+\mathbf{Z}^{n}=\mathbf{R}^{n}$ up to a measure zero set. But each $\Omega+\alpha, \alpha \in \mathbf{Z}^{n}$, is a compact set. So any $x_{0} \in \mathbf{R}^{n}$ must lie in the closure of some $\Omega+\alpha$, and hence in $\Omega+\alpha$. Thus $\Omega+\mathbf{Z}^{n}=\mathbf{R}^{n}$.

It remains to be shown that $\Omega$ has nonempty interior. Let $U$ be the closure of the unit ball $B_{1}(0)$ of $\mathbf{R}^{n}$. So

$$
U=\bigcup_{\alpha \in \mathbf{Z}^{n}}(\Omega+\alpha) \cap U
$$

Clearly $(\Omega+\alpha) \cap U \neq \emptyset$ for only finitely many $\alpha \in \mathbf{Z}^{n}$, and each $(\Omega+\alpha) \cap U$ is closed. So $U=\bigcup_{j=1}^{m} U_{i}$ where $U_{i}=\left(\Omega+\alpha_{j}\right) \cap U$. Hence at least one of the $U_{i}$ 's has nonempty interior, and so $\Omega$ has nonempty interior.

3. Proofs of Main Theorems. We first state some facts concerning nonnegative matrices, that is, matrices with nonnegative entries. We call a nonnegative matrix $B=\left[b_{i j}\right] \in M_{r}(\mathbf{R})$ irreducible if for all $1 \leq i, j \leq r$ there exist $1 \leq k_{1}, \ldots, k_{m} \leq r$ such that

$$
b_{i k_{1}} b_{k_{1} k_{2}} \cdots b_{k_{m-1} k_{m}} b_{k_{m} j}>0 \text {. }
$$

Otherwise $B$ is called reducible. $B$ is primitive if $B^{k}$ are irreducible for all $k \geq 1$. The Perron-Frobenius Theorem states that if a nonnegative $B$ is irreducible then the spectral radius $\rho=\rho(B)$ is a simple eigenvalue of $B$, and $B$ has a $\rho$-eigenvector $v$ that is positive. On the other hand, if $B$ is reducible then $B$ can be block triangularized by a permutation matrix $P$, that is

$$
P B P^{-1}=\left[\begin{array}{cc}
B_{11} & 0 \\
B_{21} & B_{22}
\end{array}\right], \quad B_{11}, B_{22} \text { are nonempty. }
$$

We now go back to the vector refinement equation

$$
f(x)=\sum_{\alpha \in \mathbf{Z}^{n}} c_{\alpha} f(A x-\alpha)
$$

As before, here $A \in M_{n}(\mathbf{Z})$ is expanding, $c_{\alpha} \in M_{r}(\mathbf{R})$ and $c_{\alpha} \neq 0$ for only finitely many $\alpha$.

Lemma 3.1. Suppose that all $c_{\alpha}$ in (3.1) are zero-one matrices and $\rho(c)=$ $|\operatorname{det}(A)|$ where $c:=\sum_{\alpha \in \mathbf{Z}^{n}} c_{\alpha}$. If $c$ is reducible then the vector refinement equation (3.1) cannot admit a Haar-type scaling function vector solution. 
Proof. Since $c$ is reducible we may assume without loss of generality that

$$
c=\left[\begin{array}{cc}
\hat{c} & 0 \\
* & \tilde{c}
\end{array}\right], \quad \hat{c} \in M_{m}(\mathbf{R}), \hat{c} \in M_{r-m}(\mathbf{R}),
$$

for some $0<m<r$. The nonnegativity forces every $c_{\alpha}$ to be of the form

$$
c_{\alpha}=\left[\begin{array}{cc}
\hat{c}_{\alpha} & 0 \\
* & \tilde{c}_{\alpha}
\end{array}\right], \quad \hat{c}_{\alpha} \in M_{m}(\mathbf{R}), \tilde{c}_{\alpha} \in M_{r-m}(\mathbf{R}) .
$$

Now assume that $\chi_{\Omega}$ is a Haar-type scaling function vector satisfying (3.1) for some $\boldsymbol{\Omega}=\left(\Omega_{1}, \ldots, \Omega_{r}\right) \in \mathcal{C}_{n}^{r}$. Let $\widehat{\boldsymbol{\Omega}}=\left(\Omega_{1}, \ldots, \Omega_{m}\right) \in \mathcal{C}_{n}^{m}$. Then

$$
\chi_{\widehat{\mathbf{\Omega}}}(x)=\sum_{\alpha \in \mathbf{Z}^{n}} \hat{c}_{\alpha} \chi_{\widehat{\mathbf{\Omega}}}(A x-\alpha) .
$$

Define $\widehat{\mathcal{D}}_{i j}=\left\{\alpha \in \mathbf{Z}^{n}:\left(\hat{c}_{\alpha}\right)_{i j}=1\right\}$ for $1 \leq i, j \leq m$. Then $\widehat{\boldsymbol{\Omega}}$ satisfies

$$
A\left(\Omega_{i}\right)=\bigcup_{j=1}^{m}\left(\Omega_{j}+\widehat{\mathcal{D}}_{i j}\right), \quad 1 \leq i \leq m .
$$

It follows from Theorem 2.4 that either $\mu\left(\cup_{i=1}^{m} \Omega_{i}\right)=0$ or $\mu\left(\cup_{i=1}^{m} \Omega_{i}\right) \geq 1$. But all $\mu\left(\Omega_{i}\right)>0$ by definition, we must thus have $\mu\left(\cup_{i=1}^{m} \Omega_{i}\right) \geq 1$. However, this forces $\mu\left(\cup_{i=1}^{r} \Omega_{i}\right)>1$ and hence the orthogonality of $\left\{\chi_{\Omega_{i}}(x-\alpha): 1 \leq i \leq r, \alpha \in \mathbf{Z}^{n}\right\}$ cannot hold, a contradiction. [

Proof of Theorem 1.1. (i) Let $\langle.,$.$\rangle denote the standard inner product in L^{2}\left(\mathbf{R}^{n}\right)$. The scaling function vector $\chi_{\Omega}$ gives

$$
\left\langle\chi_{\Omega_{i}}(x), \chi_{\Omega_{j}}(x-\alpha)\right\rangle=0, \quad i \neq j \text { or } \alpha \neq 0 .
$$

This means that $\Omega_{i}$ and $\Omega_{j}+\alpha$ are measure disjoint for $i \neq j$ or $\alpha \neq 0$. The vector refinement equation (1.6) now yields $c_{\alpha} \in M_{r}(\{0,1\})$ immediately.

(ii) By Lemma 2.1 there exists a diagonal matrix $\Lambda=\operatorname{diag}\left(\lambda_{1}, \ldots, \lambda_{r}\right)$ with all $\lambda_{i}>0$ such that

$$
\sum_{\alpha \in \mathbf{Z}^{n}} c_{\alpha+A \beta_{1}} \Lambda c_{\alpha+A \beta_{2}}^{T}=\delta_{\beta_{1}-\beta_{2}}|\operatorname{det}(A)| \Lambda
$$

It follows that for $\beta_{1} \neq \beta_{2}$ the matrices $c_{\alpha+A \beta_{1}}$ and $c_{\alpha+A \beta_{2}}$ cannot have entries of 1 in a common column. In addition, no $c_{\alpha}$ can have two or more entries of 1 in a column, or it would create a positive off-diagonal entry in $c_{\alpha} \Lambda c_{\alpha}^{T}$ to contradict (3.4). Therefore $b_{\alpha}=\sum_{\beta \in \mathbf{Z}^{n}} c_{\alpha+A \beta}$ is a zero-one matrix with at most one entry of 1 in each column.

We show that $b_{\alpha}$ cannot have a zero column. Let $\alpha_{1}, \ldots, \alpha_{q}$ be a complete residue system $(\bmod A)$, where $q=|\operatorname{det}(A)|$. Note that

$$
\begin{aligned}
\sum_{j=1}^{q} b_{\alpha_{j}} \Lambda b_{\alpha_{j}}^{T} & =\sum_{j=1}^{q} \sum_{\beta_{1} \in \mathbf{Z}^{n}} \sum_{\beta_{2} \in \mathbf{Z}^{n}} c_{\alpha_{j}+A \beta_{1}} \Lambda c_{\alpha_{j}+A \beta_{2}}^{T} \\
& =\sum_{\gamma \in \mathbf{Z}^{n}} \sum_{\alpha \in \mathbf{Z}^{n}} c_{\alpha} \Lambda c_{\alpha+A \gamma}^{T} \\
& =q \Lambda .
\end{aligned}
$$


Now assume that some $b_{\alpha_{i}}$, say $b_{\alpha_{1}}$, has a zero column. Then for $v=[1, \ldots, 1]^{T}$ we have

$$
v^{T} b_{\alpha_{1}} \Lambda b_{\alpha_{1}}^{T} v<\sum_{i=1}^{r} \lambda_{i}
$$

Therefore

$$
v^{T} q \Lambda v=\sum_{i=1}^{r} v^{T} b_{\alpha_{i}} \Lambda b_{\alpha_{i}}^{T} v<q \sum_{i=1}^{r} \lambda_{i}
$$

contradicting $v^{T} q \Lambda v=q \sum_{i=1}^{r} \lambda_{i}$. So $b_{\alpha_{i}}$ has no zero column. Now for any $\alpha \in \mathbf{Z}^{n}$ we have $b_{\alpha}=b_{\alpha_{i}}$ for some $i$. This proves (ii).

(iii) This is Lemma 3.1 .

(iii') Iterating (1.6) yields

$$
\chi_{\boldsymbol{\Omega}}(x)=\sum_{\alpha \in \mathbf{Z}^{n}} \sum_{\beta \in \mathbf{Z}^{n}} c_{\alpha} c_{\beta} \chi_{\boldsymbol{\Omega}}\left(A^{2} x-A \alpha-\beta\right) .
$$

For the new equation, $\sum_{\alpha \in \mathbf{Z}^{n}} \sum_{\beta \in \mathbf{Z}^{n}} c_{\alpha} c_{\beta}=c^{2}$. By (iii) $c^{2}$ is irreducible. By the same argument, all $c^{k}$ are irreducible. Hence $c$ is primitive.

(vi) The measure disjointness of $\left\{\Omega_{1}, \ldots, \Omega_{r}\right\}$ is clear. Now by Theorem 2.4 the set $\Omega:=\cup_{i=1}^{r} \Omega_{i}$ has the property that $\Omega+\mathbf{Z}^{n}=\mathbf{R}^{n}$. But the orthogonality condition forces $\mu(\Omega) \leq 1$. Hence $\mu(\Omega)=1$ and therefore $\mathbf{R}^{n}=\Omega+\mathbf{Z}^{n}$ is a tiling.

REMARK. In general $\Omega:=\cup_{i=1}^{r} \Omega_{i}$ is not itself a self-affine tile. It is not hard to check that $\Omega$ is a self-affine tile if and only if $\cup_{i=1}^{r} \mathcal{D}_{i j}=\mathcal{D}$ for all $1 \leq j \leq r$, where $\mathcal{D} \in \mathbf{Z}^{n}$ with $|\mathcal{D}|=|\operatorname{det}(A)|$ and $\mathcal{D}_{i j}=\left\{\alpha:\left(c_{\alpha}\right)_{i j}=1\right\}$. We omit the proof here.

LEMMA 3.2. Let $D_{1}, \ldots, D_{m}$ be measure disjoint compact sets in $\mathbf{R}^{n}$ such that $\cup_{i=1}^{m} \frac{D_{i}}{D_{i}}=\overline{B_{K}\left(x_{0}\right)}$. Then for any $D_{i}$ having positive Lebesgue measure we have $D_{i}^{o} \neq \emptyset$ and $\overline{D_{i}^{o}}=D_{i}$ up to a measure zero set.

Proof. Suppose that some $D_{i}$, say $D_{1}$, has positive Lebesgue measure but no interior point. Then for any $y \in D_{1}$ there exists a sequence $\left\{y_{k}\right\}$ such that $\lim _{k \rightarrow \infty} y_{k}=y$ and $y_{k} \notin D_{1}$. Hence $y \in \cup_{i=2}^{m} D_{i}$, and so $D_{1} \subseteq \cup_{i=2}^{m} D_{i}$. This contradicts the measure disjointness of $D_{i}$ 's. Thus $D_{i}^{o} \neq \emptyset$ for all $D_{i}$ with positive Lebesgue measure. Now using the same argument we have $\partial D_{i} \subseteq \cup_{j \neq i} D_{j}$. Hence by measure disjointness again $\mu\left(\partial D_{i}\right)=0$ for all $i$. Thus $D_{i}=\overline{D_{i}^{o}}$ up to a measure zero set.

Proof of Theorem 1.2. The assumed condition (ii) implies that the sum of the entries in each column of $c$ is $q=|\operatorname{det}(A)|$. So the nonnegative matrix $m(0)=q^{-1} c$ is column stochastic. Therefore $\rho(m(0))=1$. By Theorem 2.2 there exists a compactly supported $f(x)=\left[f_{1}(x), \ldots, f_{r}(x)\right]^{T}, f(x) \neq 0$ and each $f_{i}(x) \in L^{2}\left(\mathbf{R}^{n}\right)$, such that

$$
f(x)=\sum_{\alpha \in \mathbf{Z}^{n}} c_{\alpha} f(A x-\alpha)
$$

Let $\mathbf{U}=\left(U_{1}, \ldots, U_{r}\right)$ where each $U_{i}$ is the essential support of $f_{i}(x)$. The vector refinement equation (1.9) implies that

$$
U_{i} \subseteq \bigcup_{j=1}^{r} A^{-1}\left(U_{j}+\mathcal{D}_{i j}\right), \quad 1 \leq i \leq r
$$


where $\mathcal{D}_{i j}=\left\{\alpha:\left(c_{\alpha}\right)_{i j}=1\right\}$. It follows by iterating (3.5) while replacing $U_{i}$ in the equation by its closure that $U_{i} \subseteq \Omega_{i}$, where $\boldsymbol{\Omega}=\left(\Omega_{1}, \ldots, \Omega_{r}\right) \in \mathcal{C}_{n}^{r}$ is the unique solution to

$$
A\left(\Omega_{i}\right)=\bigcup_{j=1}^{r}\left(\Omega_{j}+\mathcal{D}_{i j}\right), \quad 1 \leq i \leq r .
$$

Since $f \neq 0$ at least one of the $U_{i}$ 's has positive Lebesgue measure. It follows from Theorem 2.4 that $\Omega=\cup_{i=1}^{r} \Omega_{i}$ has nonempty interior.

We show that $\chi_{\Omega}$ satisfies the vector refinement equation (1.9). Note that condition (ii) implies that $\sum_{i=1}^{r}\left|\mathcal{D}_{i j}\right|=|\operatorname{det}(A)|$. Therefore

$$
\sum_{i=1}^{r} \mu\left(\bigcup_{j=1}^{r}\left(\Omega_{j}+\mathcal{D}_{i j}\right)\right) \leq \sum_{i=1}^{r} \sum_{j=1}^{r}\left|\mathcal{D}_{i j}\right| \mu\left(\Omega_{i}\right)=|\operatorname{det}(A)| \sum_{i=1}^{r} \mu\left(\Omega_{i}\right) .
$$

But

$$
|\operatorname{det}(A)| \sum_{i=1}^{r} \mu\left(\Omega_{i}\right)=\sum_{i=1}^{r} \mu\left(A\left(\Omega_{i}\right)\right)=\sum_{i=1}^{r} \mu\left(\bigcup_{j=1}^{r}\left(\Omega_{j}+\mathcal{D}_{i j}\right)\right) .
$$

Thus all inequalities in (3.7) are equalities, and so all unions in $\bigcup_{j=1}^{r}\left(\Omega_{j}+\mathcal{D}_{i j}\right)$ are measure disjoint. This forces

$$
\chi_{\boldsymbol{\Omega}}(x)=\sum_{\alpha \in \mathbf{Z}^{n}} c_{\alpha} \chi_{\boldsymbol{\Omega}}(A x-\alpha) .
$$

Note that the vector $v=\int_{\mathbf{R}^{n}} \chi_{\boldsymbol{\Omega}}(x) d x$ is a $|\operatorname{det}(A)|$-eigenvector of $c$. It follows from the irreducibility of $c$ that $v$ is a positive vector. So all $\mu\left(\Omega_{i}\right)>0$.

We now prove property (B). $\Omega^{o} \neq \emptyset$ so $\Omega_{i}^{o} \neq \emptyset$ for some $i$, say $\Omega_{1}^{o} \neq \emptyset$. Since $A$ is expanding, for some sufficiently large $N>0$ the set $A^{N}\left(\Omega_{1}\right)$ contains an interior point $x_{0} \in \mathbf{Z}^{n}$. So 0 is an interior point of $\tilde{\Omega}_{1}:=A^{N}\left(\Omega_{1}\right)-x_{0}$. This means that for each $k>0$ there exists an $m_{k}>0$ such that the ball $B_{k}(0)$ is covered by $A^{m_{k}}\left(\tilde{\Omega}_{1}\right)$. It follows from the inflation property (3.6) that there exist finite subsets $\mathcal{J}_{1}^{k}, \ldots, \mathcal{J}_{r}^{k}$ of $\mathbf{Z}^{n}$ such that

$$
B_{k}(0) \subseteq \bigcup_{i=1}^{r}\left(\Omega_{i}+\mathcal{J}_{i}^{k}\right)
$$

where all unions in (3.9) are measure disjoint. Now for any given $K>0$ and each $i$ there are only finitely many distinct $\mathcal{J}_{i}^{k} \cap B_{K}(0)$. So we can find a subsequence $\mathcal{J}_{1}^{m_{k}}, \ldots, \mathcal{J}_{r}^{m_{k}}$ such that $\mathcal{J}_{i}^{m_{k}} \rightarrow \mathcal{J}_{i}$ in the sense that for each $K>0, \mathcal{J}_{i}^{m_{k}} \cap B_{K}(0)=$ $\mathcal{J}_{i} \cap B_{K}(0)$ for sufficiently large $m_{k}$. By (3.9)

$$
\mathbf{R}^{n}=\bigcup_{i=1}^{r}\left(\Omega_{i}+\mathcal{J}_{i}\right)
$$

with all unions measure disjoint, proving (B).

To prove (A), take a sufficiently large $K>0$ so that $\overline{B_{K}(0)}$ contains at least one translate of each $\Omega_{i}$ in the tiling (3.10). So $\overline{B_{K}(0)}$ is the measure disjoint union of compact sets that include translates of each $\Omega_{i}$. Since all $\Omega_{i}$ have positive Lebesgue measure, by Lemma $3.2 \Omega_{i}$ all have nonempty interior and $\Omega_{i}=\overline{\Omega_{i}^{o}}$ up to a measure zero set. This proves $(\mathrm{A})$. 
4. Examples. We now look at some examples of self-affine multi-tiles and Haartype scaling function vectors. We first select an expanding integer matrix $A$, and then choose coefficient matrices $c_{\alpha}$ satisfying properties (i)-(iii) of Theorem 1.1. In Examples 1-3 our expanding matrix $A$ is the quincunx matrix (also refered to as the "dragon" matrix),

$$
A=\left[\begin{array}{cc}
1 & 1 \\
-1 & 1
\end{array}\right]
$$

ExAmPle 1. We let $A$ be the quincunx matrix and choose the coefficient matrices to be

$$
c_{(0,0)}=\left[\begin{array}{ll}
1 & 1 \\
0 & 0
\end{array}\right], \quad c_{(1,0)}=\left[\begin{array}{ll}
0 & 0 \\
0 & 1
\end{array}\right], \quad c_{(0,1)}=\left[\begin{array}{ll}
0 & 0 \\
1 & 0
\end{array}\right] .
$$

The self-affine multi-tile $\boldsymbol{\Omega}=\left(\Omega_{1}, \Omega_{2}\right)$ is shown in Figure 1 .

ExAmple 2. We again choose $A$ to be the quincunx matrix and $r=3$. The coefficient matrices are

$$
c_{(0,0)}=\left[\begin{array}{lll}
0 & 1 & 0 \\
0 & 0 & 0 \\
1 & 0 & 1
\end{array}\right], \quad c_{(1,0)}=\left[\begin{array}{lll}
0 & 0 & 0 \\
1 & 1 & 0 \\
0 & 0 & 0
\end{array}\right], \quad c_{(0,1)}=\left[\begin{array}{lll}
0 & 0 & 1 \\
0 & 0 & 0 \\
0 & 0 & 0
\end{array}\right] .
$$

The self-affine multi-tile $\boldsymbol{\Omega}=\left(\Omega_{1}, \Omega_{2}, \Omega_{3}\right)$ is shown in Figure 2 .

EXAmple 3. We let $A$ be the quincunx matrix and $r=4$. The coefficient matrices are chosen as

$$
c_{(0,0)}=\left[\begin{array}{llll}
1 & 1 & 0 & 0 \\
0 & 0 & 1 & 0 \\
0 & 0 & 0 & 1 \\
0 & 0 & 0 & 0
\end{array}\right], c_{(1,0)}=\left[\begin{array}{llll}
0 & 0 & 1 & 0 \\
0 & 0 & 0 & 0 \\
0 & 0 & 0 & 0 \\
0 & 0 & 0 & 1
\end{array}\right], c_{(0,1)}=\left[\begin{array}{llll}
0 & 0 & 0 & 0 \\
0 & 0 & 0 & 0 \\
1 & 0 & 0 & 0 \\
0 & 1 & 0 & 0
\end{array}\right] .
$$

$\boldsymbol{\Omega}=\left(\Omega_{1}, \Omega_{2}, \Omega_{3}\right)$ is shown in Figure 3 .

EXAMPLE 4. In this last example we let

$$
A=\left[\begin{array}{cc}
2 & 1 \\
-1 & 1
\end{array}\right]
$$

which has $\operatorname{det}(A)=3$. We choose the coefficient matrices to be

$$
c_{(0,0)}=\left[\begin{array}{ll}
1 & 0 \\
0 & 1
\end{array}\right], c_{(0,-1)}=\left[\begin{array}{ll}
0 & 0 \\
1 & 0
\end{array}\right], c_{(1,-1)}=\left[\begin{array}{ll}
1 & 1 \\
0 & 0
\end{array}\right], c_{(1,0)}=\left[\begin{array}{ll}
0 & 1 \\
0 & 0
\end{array}\right] \text {. }
$$

$\boldsymbol{\Omega}=\left(\Omega_{1}, \Omega_{2}\right)$ is shown in Figure 4 .

We have obtained these examples somewhat by trial and error, by generating the plots of the tiles, and choosing ones that looked nice. In some cases the plots of the different tiles were very dispersed, and hard to visualize. There are many other possibilities for Haar-type scaling function vectors, using other dilation matrices $A$. 


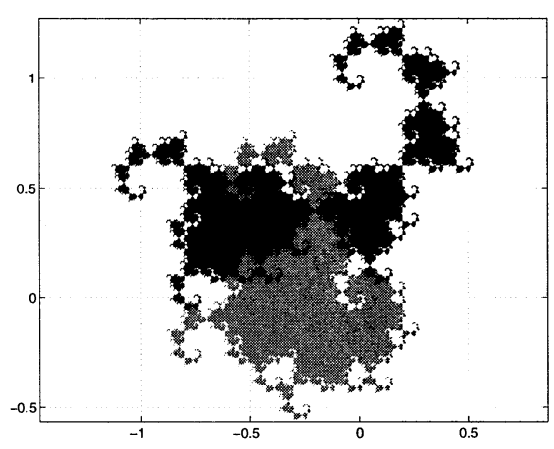

Figure 1: $\Omega_{1}($ light $), \Omega_{2}($ dark $)$

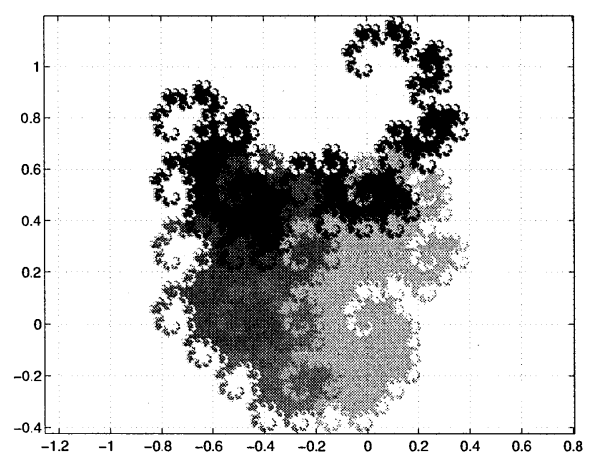

Figure 3: $\Omega_{1}, \Omega_{2}, \Omega_{3}, \Omega_{4},($ light $\ldots$ dark $)$

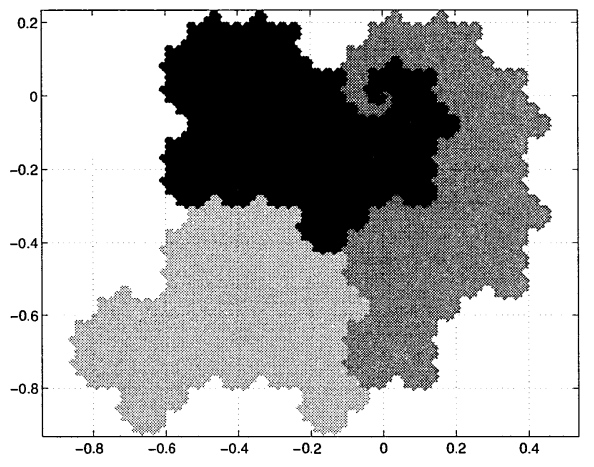

Figure 2: $\Omega_{1}, \Omega_{2}, \Omega_{3}($ light ...dark $)$

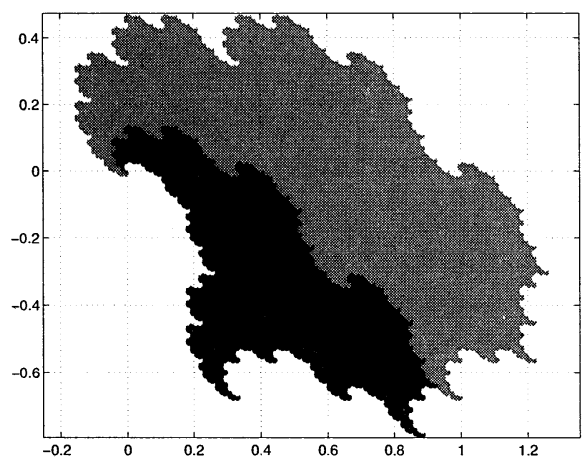

Figure 4: $\Omega_{1}($ light $), \Omega_{2}($ dark $)$ 


\section{REFERENCES}

[1] C. BANDT, Self-similar sets 5. Integer matrices and fractal tilings of $R^{n}$, Proc. Amer. Math Soc., 112 (1991), pp. 549-562.

[2] M. BARnsley, Fractals everywhere, Academic Press, 1988.

[3] M. A. BERGER AND Y. WANG, Multidimensional two-scale dilation equations, in Wavelets-A Tutorial in Theory and Applications, C. K. Chui, ed., 1992, pp. 295-323.

[4] A. Cohen, I. Daubechies, and G. Plonka, Regularity of refinable function vectors, J. Fourier Anal. Appl., 3 (199'7), pp. 295-324.

[5] I. Daubechies, Ten Lectures on Wavelets, SIAM, Philadelphia, PA, 1992.

[6] G. Donovan, J. Geronimo, D. Hardin, And P. Massopust, Construction of orthogonal wavelets using fractal interpolation functions, SIAM J. Math. Anal., 27 (1996), pp. 11581192.

[7] K. J. FAlconer, The Geometry of Fractal Sets, Cambridge Univ. Press, Cambridge, 1985.

[8] J. Geronimo AND D. HARdin, Fractal interpolation surfaces and a related 2-D multiresolution analysis, J. Math. Anal. Appl., 176 (1993), pp. 561-586.

[9] J. Geronimo, D. Hardin, And P. Massopust, Fractal functions and wavelet expansions based on several scaling functions, J. Approx. Th., 78 (1994), pp. 373-401.

[10] T. Goodman and S. L. LeE, Wavelets of multiplicity r, Trans. Amer. Math. Soc., 342 (1994), pp. 307-324.

[11] K. Gröchenig And A. HaAs, Self-similar lattice tilings, J. Fourier Analysis, 1 (1994), pp. 131-170.

[12] K. Gröchenig, A. HaAs, And A. Raugi, Self-affine tilings with several tiles I, Preprint.

[13] K. GRöChEnig AND W. MAdYCH, Multiresolution analysis, Haar bases, and self-similar tilings, IEEE Trans. Inform. Th., 38:2, Part 2 (1992), pp. 558-568.

[14] B. Grünbaum and G. C. Shephard, Tilings and Patterns, Freeman, New York, 1987.

[15] D. HARDin, B. KesSler, AND P. MASSOPUST, Multiresolution analysis based on fractal functions, J. Approx. Th., 71 (1992), pp. 373-401.

[16] C. Heil AND D. Collela, Matrix refinement equations: existence and uniqueness, J. Fourier Anal. Appl., 2 (1996), pp. 363-377.

[17] J. E. Hutchinson, Fractals and self-similarity, Indiana Univ. Math. J., 30 (1981), pp. $713-747$.

[18] R. KenYon, Self-replicating tilings, in Symbolic Dynamics and Applications, P. Walters, ed., Contemporary Math., 135, AMS, Providence, RI, 1992, pp. 239-264.

[19] J. C. Lagarias ANd Y. Wang, Self-affine tiles in $\mathbf{R}^{n}$, Adv. in Math., 121 (1996), pp. 21-49.

[20] J. C. LAGARIAS AND Y. WANG, Integral self-affine tiles in $\mathbf{R}^{n}$ I. Standard and nonstandard digit sets, J. London Math. Soc., 53 (1996), pp. 161-179.

[21] J. C. LAGARIAS AND Y. WANG, Integral self-affine tiles in $\mathbf{R}^{n}$, part II: lattice tilings, J. Fourier Anal. and Appl., 3 (1997), pp. 83-101.

[22] J. C. LAgARIAS AND Y. WANG, Haar bases in $\mathbf{R}^{n}$ and algebraic number theory, J. Number Theory, 57 (1996), pp. 181-197.

[23] J. C. LAGARIAS AND Y. WANG, Haar type orthonormal wavelet basis in $\mathbf{R}^{2}$, J. Fourier Analysis and Appl., 2 (1995), pp. 1-14.

[24] W. LAWTON, Necessary and sufficient conditions for constructing orthonormal wavelet bases, J. Math. Phys., 32 (1991), pp. 57-61.

[25] W. LAWTon, S. L. LeE, AND Z. SHen, An algorithm for matrix extension and wavelet construction, Math. Comp., 65, pp. 723-737.

[26] S. Mallat, Multiresolution analysis and wavelets, Tans. Amer. Math. Soc., 315 (1989), pp. 69-88.

[27] D. MAUldin AND S. Williams, Hausdorff dimension in graph direct constructions, Trans. Amer. Math. Soc., 309 (1988), pp. 811-829.

[28] R. S. Strichartz, Wavelets and Self-Affine Tilings, Constructive Approximation, 9 (1993), pp. 327-346.

[29] R. S. Strichartz, Self-similarity in harmonic analysis, J. Fourier Anal. Appl., 1 (1994), pp. $1-37$.

[30] W. P. Thurston, Groups, Tilings and Finite State Automata, AMS Colloquium Lect. Notes, 1989.

[31] A. VINCE, Replicating tessellations, SIAM J. Discrete Math., 3 (1993), pp. 501-521. 